\title{
Multi-photon absorption in optical gratings for matter waves
}

\author{
Kai Walter ${ }^{1}$, Stefan Nimmrichter ${ }^{2}$, and Klaus Hornberger ${ }^{1}$ \\ ${ }^{1}$ University of Duisburg-Essen, Faculty of Physics, Lotharstraße 1-21, 47048 Duisburg, Germany \\ ${ }^{2}$ Centre for Quantum Technologies, National University of Singapore, 3 Science Drive 2, Singapore 117543
}

\begin{abstract}
We present a theory for the diffraction of large molecules or nanoparticles at a standing light wave. Such particles can act as a genuine photon absorbers due to their numerous internal degrees of freedom effecting fast internal energy conversion. Our theory incorporates the interplay of three light-induced properties: the coherent phase modulation due to the dipole interaction, a non-unitary absorption-induced amplitude modulation described as a generalized measurement, and a coherent recoil splitting that resembles a quantum random walk in steps of the photon momentum. We discuss how these effects show up in near-field and far-field interference schemes, and we confirm our effective description by a dynamic evaluation of the grating interaction, which accounts for the internal states.
\end{abstract}

PACS numbers: 03.75.Dg, 03.65.Yz

\section{INTRODUCTION}

Exploring matter-wave interference with heavy molecules and nanoparticles is of fundamental interest, as it allows exploring the possible limits of quantum mechanics at macroscopic scales [1, 2], and developing new tools to measure accurately internal molecular properties [3].

Motivated by experiments [3 9] that use a standing light field as diffraction element, we focus on the interaction between a standing-wave laser grating and a delocalized and internally complex molecule. It has been seen earlier that spontaneous emission, light scattering, or thermal radiation induced by an optical grating can lead to decoherence [10 12]. However, complex molecules tend to absorb the light from the laser grating predominantly without subsequent reemission because they can rapidly redistribute the photon energy to many internal states, acting effectively as an energy sink. This applies all the more so to large, optically levitated nanoparticles, e.g. silica spheres, which are candidates for novel optomechanics and matter-wave interference schemes [13 16]. It is intriguing to ask whether such molecules are capable to interfere after the absorption given that it might reveal "which-way" information and the position of the molecular center of mass. In the following we discuss how the molecules are still able to interfere even after absorption of many laser photons.

Specifically, the theory developed below is required to quantitatively describe a recent experiment with $\mathrm{C}_{70}$ fullerenes which featured a high laser power and unprecedented velocity resolution [9]. It provides evidence that the momentum recoil upon photon absorption from a standing light field is coherent in the sense that it leads to a superposition of the momentum kicks associated with the two possible photon directions, rather than to a mixture. Despite the state-insensitive detection and hence the lack of coherence in the number of absorptions, the experiment rules out a classical random-walk description for the absorption process.

The structure of this article is as follows. In Sect. II we make use of the formalism of generalized measurements [17 21] to describe all relevant aspects of the lightparticle interaction: the influence of the dipole force, the stochastic impact of photon absorptions, and the matter-wave amplitude modulation associated with the absorption-induced postselection. The influence of these effects on matter-wave interferometry is then discussed in Sect. III by considering near-field and far-field schemes. In the near-field case, photon absorption modulates the periodic fringe pattern and lowers the interference visibility on average, while in the far-field case, photon absorption leads to new features in the observed interferogram. The nonclassical nature of the absorption recoil is only visible in the near field. In Sect. IV we develop dynamic models of the laser-grating interaction in order to corroborate and generalize the measurement-based description of Sect. [I. A ladder model for the internal state allows evaluating the center-of-mass quantum dynamics in presence of absorption, accounting for possible photo-induced changes of the optical molecular properties. Moreover, we consider a three-level model with a dark state in order to incorporate partially coherent Rabi-oscillations expected for resonant transitions. We present our conclusions in Sect. V.

\section{OPTICAL GRATING TRANSFORMATION AS A GENERALIZED MEASUREMENT}

We start by developing an effective measurement-based description of the interaction between a standing-wave laser grating and an absorbing molecule in terms of a generalized measurement transformation. It serves to incorporate the essential effect of photon absorption on the center-of-mass motion of complex molecules in the absence of detailed knowledge about the molecule's level structure and transition dipole moments. Avoiding a microscopic treatment, we model the interaction effectively by means of the particle's complex susceptibility including the dipole polarizability and the absorption cross section at a given laser wavelength. Our approximate 
model would fail if the life times of the electrically excited states were long compared with the interaction time, see Sect. IVC For large molecules the relaxation time is typically on the order of picoseconds so that our effective description should be valid. For even larger nanoparticles, the optical response is often entirely characterized by a phenomenological dielectric function, which may exhibit broad internal (plasmonic) resonances at optical-to-UV wavelengths [22].

The model for the scattering of the molecular centerof-mass motion off the standing light field comprises two independent steps: On the one hand, a unitary state transformation describes the coherent matter-wave phase modulation due to the dipole interaction, see Sect. [IA On the other hand, the non-unitary transformation developed in Sect. IIB accounts for the change of state due to photon absorption. In the context of interferometry, it is convenient to represent the motional quantum state of the molecular center of mass in phase space. We therefore formulate the grating transformation in terms of the Wigner function [23] in Sect. IIC The present formalism applies to all experimental scenarios where nanoparticles interact with optical standing waves in the Raman-Nath, or short-time, regime.

\section{A. Phase modulation}

A sub-wavelength molecule or nanoparticle interacts with off-resonant light fields mainly through its frequency-dependent dipole polarizability $\alpha_{\text {SI }}$. The particle is then subject to the dipole force proportional to the gradient of the local time-averaged standing-wave light intensity,

$$
I(x, y, z)=\frac{8 P}{\pi w_{y} w_{z}} \exp \left(-\frac{2 y^{2}}{w_{y}^{2}}-\frac{2 z^{2}}{w_{z}^{2}}\right) \cos ^{2}\left(k_{\mathrm{L}} x\right) .
$$

Here, $x$ denotes the standing-wave axis, while $k_{\mathrm{L}}, w_{y}, w_{z}$ and $P$ are the wave number, the waists, and the runningwave power of a retro-reflected gaussian laser beam forming the standing wave. In the Raman-Nath regime 24] of a short interaction time and high kinetic energy, when the molecule rapidly crosses the laser beam at an approximately constant velocity $v_{z}$ in the $x z$-plane, the dipole interaction is captured by the time-dependent potential $V(x, t)=-\left(2 \pi \alpha_{\mathrm{SI}} / 4 \pi \varepsilon_{0} c\right) I\left(x, 0, v_{z} t\right)$. If also the transverse motion along $x$ and $y$ can be neglected during the passage, the scattering problem effectively reduces to one dimension. The molecule acquires an $x$-dependent phase [25, 26] that results in a unitary scattering transformation,

$$
\mathrm{U}=\exp [i \phi(\mathrm{x})]=\int d x \exp [i \phi(x)]|x\rangle\langle x|,
$$

with $x$ the one-dimensional position operator in the standing-wave direction. This position-dependent phase shift $\phi(x)$ is given by the eikonal action accumulated during passage, i.e. by the time integral of the interaction potential,

$$
\begin{aligned}
\phi(x) & =-\frac{1}{\hbar} \int_{-\infty}^{\infty} d t V(x, t)=\phi_{0} \cos ^{2}\left(k_{\mathrm{L}} x\right), \\
\phi_{0} & =\frac{2 \sqrt{2}}{\sqrt{\pi} \varepsilon_{0}} \frac{\alpha_{\mathrm{SI}}}{\hbar c} \frac{P}{w_{y} v_{z}} .
\end{aligned}
$$

For realistic sub-wavelength molecules or nanoparticles, corrections to this simple scattering model can be attributed to their anisotropy and to photon absorption. We focus on the latter effect in the following. For highly anisotropic molecules, described in terms of a polarizability tensor, the fast molecular rotations will lead to phase averaging and thus to degraded interference [27].

\section{B. Photon absorption}

Standing light waves have often been employed as pure phase gratings [6, 28 34]. In the case of atoms and small molecules, the absorptionless phase-grating regime is achieved by detuning the laser wavelength sufficiently from internal resonances. The treatment of large molecules and nanoparticles requires a different approach since the numerous rovibrational degrees of freedom give rise to a landscape of broad (collective) resonances in the absorption spectrum $\sigma_{\mathrm{abs}}(\omega)$. The linear response of the molecule to the field is described by a complex susceptibility that accounts for both the dipole interaction and the absorption, $\chi=\alpha_{\mathrm{SI}}+i c \varepsilon_{0} \sigma_{\mathrm{abs}} / \omega_{\mathrm{L}}$ [35]. Photon absorption is negligible as long as $\beta=\operatorname{Im}(\chi) / \operatorname{Re}(\chi) \ll 1$.

For lack of a microscopic description, we base our absorption model solely on the knowledge of collective properties that can be measured independently, such as the absorption cross-section $\sigma_{\text {abs }}$ and the heat capacity $C$, as well as on the assumption of an internal heat sink: The excess energy $\hbar \omega_{L}$ of an absorbed photon is assumed to be "dissipated" immediately, i.e. redistributed among the many internal degrees of freedom. This results in a mean increase of the internal microcanonical temperature by $\Delta T=\hbar \omega_{\mathrm{L}} / C$. In practice, this turns out to be an excellent approximation for many complex molecules and nanoparticles with $C / k_{\mathrm{B}} \gg 1$, as long as only few photons are absorbed and the particle does not heat up too much. In specific cases, it might be necessary to take a radiative reemission of internal excess energy into account, either by fluorescence or by thermal black-body radiation at high temperatures. This would result in additional decoherence, the theoretical description of which can be found elsewhere [12, 36].

From the point of view of operational quantum mechanics [37], photon absorption can be described as a generalized measurement transformation [17-21], as specified by a set of measurement operators $\left\{M_{\ell}\right\}$ with $\sum_{\ell=0}^{\infty} \mathrm{M}_{\ell} \mathrm{M}_{\ell}^{\dagger}=\mathbb{1}$. In this framework, the number $\ell=$ $0,1,2, \ldots$ of absorbed photons, as recorded by the excess 
energy of the internal degrees of freedom corresponds to the measurement result. In principle, this thermal encoding can be revealed by a calorimetric absorption detection scheme, which yields the number $\ell$ of absorbed photons by measuring their internal energy. When the measurement indicates the absorption of $\ell$ photons the conditional transformation of the reduced center-of-mass state $\rho$ reads

$$
\rho \rightarrow \rho_{\ell}:=\frac{\mathrm{M}_{\ell} \rho \mathrm{M}_{\ell}^{\dagger}}{P_{\ell}(\rho)}, \quad P_{\ell}(\rho)=\operatorname{tr}\left[\mathrm{M}_{\ell}^{\dagger} \mathrm{M}_{\ell} \rho\right]
$$

Considering the particle-laser interaction in the RamanNath regime, i.e. in the limit of the short interaction time, the transverse motion between two subsequent absorption events can be neglected. This implies that the measurement operators $\mathrm{M}_{\ell}$ are diagonal in position, which ensures that the absorption probability depends only on the spatial probability distribution, $P_{\ell}(\rho)=$ $\int d x p_{\ell}(x)\langle x|\rho| x\rangle$. Here, $p_{\ell}(x)=\left|M_{\ell}(x)\right|^{2}$ represents the position dependent absorption probability. The measurement operator is therefore of the form

$$
\mathrm{M}_{\ell}=\int d x M_{\ell}(x)|x\rangle\left\langle x\left|=\mathrm{U}_{\ell}\right| \mathrm{M}_{\ell}(\mathrm{x})\right|
$$

with a unitary, position-dependent phase $\mathrm{U}_{\ell}=$ $\exp \left[i \phi_{\ell}(\mathrm{x})\right]$, which does not change the absorption probability $p_{\ell}(x)$. The choice of the unitary is not arbitrary; it must be consistent with the coherent phase modulation mediated by the molecular polarizability, as discussed in the previous section. Moreover, since the standingwave field can be seen as a superposition of two counterpropagating plane wave modes with wave vectors $k_{\mathrm{L}} \mathbf{e}_{x}$ and $-k_{\mathrm{L}} \mathbf{e}_{x}$, we expect that a superposition of recoils with momenta $\hbar k_{\mathrm{L}}$ and $-\hbar k_{\mathrm{L}}$ is coherently transferred to the particle upon absorption of a standing-wave photon. In particular, a relative phase appears between positions of a half-wavelength distances. If one photon is absorbed the diagonal elements must be proportional to $M_{1}(x) \propto \cos \left(k_{\mathrm{L}} x\right)$ because the momentum transfer of $\pm \hbar k_{\mathrm{L}}$ is described by the operator $\exp \left( \pm i k_{\mathrm{L}} \mathrm{x}\right)$. Thus for $\ell$ subsequent photon absorptions the diagonal elements of the measurement operator are $M_{\ell}(x) \propto \cos ^{\ell}\left(k_{\mathrm{L}} x\right)$, the zeros taking account for the fact that absorption does not take place at the nodes of the standing wave.

As a second step, we assume that the absorption crosssection and the polarizability do not change appreciably upon absorption, i.e. that the grating interaction does not depend on the internal state of the molecule or nanoparticle. As discussed in Sect. IV this is a reasonable approximation for low laser powers; a generalization to the case of state-dependent internal properties is also presented in Sect. IV] The assumption of state independence implies that the number $\ell$ of absorbed photons follows a Poisson distribution, $p_{\ell}(x)=e^{-n(x)} n^{\ell}(x) / \ell$ !, with a mean value of

$$
\begin{aligned}
n(x) & =\int_{-\infty}^{\infty} d t \frac{\sigma_{\mathrm{abs}}}{\hbar \omega_{\mathrm{L}}} I\left(x, 0, v_{z} t\right)=n_{0} \cos ^{2}\left(k_{\mathrm{L}} x\right), \\
n_{0} & =\frac{8}{\sqrt{2 \pi}} \frac{\sigma_{\mathrm{abs}}}{\hbar \omega_{\mathrm{L}}} \frac{P}{w_{y} v_{z}} .
\end{aligned}
$$

For the measurement operators (6) this means that the unitary part is independent of $\ell, \phi_{\ell}(x)=\phi(x)$, as given by the dipole interaction term (4), and

$$
M_{\ell}(x)=\sqrt{\frac{n_{0}^{\ell}}{\ell !}} \cos ^{\ell}\left(k_{\mathrm{L}} x\right) \exp \left(i \phi(x)-\frac{n(x)}{2}\right) .
$$

The conditional state transformation (5) then describes the diffraction of particles after the absorption of $\ell$ photons in the standing-wave grating. In principle, these particles could be postselected in a detector sensitive to the internal energy. When the detector is insensitive to the internal state, one must average over all possible absorption numbers and apply the unconditional Kraus map [21]

$$
\rho \rightarrow \rho^{\prime}=\sum_{\ell=0}^{\infty} P_{\ell}(\rho) \rho_{\ell}=\sum_{\ell=0}^{\infty} \mathbf{M}_{\ell} \rho \mathbf{M}_{\ell}^{\dagger} .
$$

As we will see in Sect. IV this transformation is consistent with the solution of a dynamical model for photon absorption.

In practice, the Poisson absorption model yields instructive, mostly analytic results for the relevant states of the incident particles, as well as for the final interferograms in both the near field and the far field. Yet, it is known from molecular spectroscopy that the absorption cross section can grow after photon absorption and intersystem crossing [38, 39]. The excited-state polarizability might differ as well, and we will incorporate these effects in Sect. IVB It turns out that such complications play only a minor quantitative role in the regime of low average absorption, $n_{0} \lesssim 2$.

In order to illustrate the influence of absorption on matter-wave diffraction, let us examine how the measurement operators act on a momentum eigenstate $|p\rangle$ of the molecule, i.e. when a coherent plane matter wave hits the grating. The phase modulation (2)-(4) leads to diffraction peaks separated by the grating momentum $2 \hbar k_{\mathrm{L}}[9]$,

$$
\mathrm{U}|p\rangle=e^{i \phi_{0} / 2} \sum_{\nu=-\infty}^{\infty} I_{\nu}\left(i \frac{\phi_{0}}{2}\right)\left|p+2 \nu \hbar k_{\mathrm{L}}\right\rangle .
$$

which follows from a Fourier decomposition of (2). The Fourier components are the modified Bessel functions $I_{\nu}(x)=i^{-\nu} J_{\nu}(i x)$. This constitutes the ideal phasegrating effect for transparent particles with $\sigma_{\mathrm{abs}}=0$. If however, $\sigma_{\mathrm{abs}}>0$, but no photon is absorbed, we must apply the measurement operator

$$
\begin{aligned}
\mathrm{M}_{0}(\mathrm{x})|p\rangle & =e^{i \phi_{0} / 2-n_{0} / 4} \sum_{\nu=-\infty}^{\infty} I_{\nu}\left(i \phi_{0} / 2-n_{0} / 4\right) \\
& \times\left|p+2 \nu \hbar k_{\mathrm{L}}\right\rangle
\end{aligned}
$$


and a different interferogram would be observed. Note that the particle then gets diffracted even for $\phi_{0}=0$. This additional source of diffraction is related to the conditional modulation of the matter-wave amplitude: The spatial density of the post-measurement state is redistributed towards the standing-wave nodes where it is more likely that no absorption took place. This conditional transformation is used to describe diffraction at optical depletion gratings [40 43], where only those particles arrive at the detector that have not absorbed any photon.

In the case of $\ell$ subsequent absorption processes, the conditional transformation is given by

$$
\begin{aligned}
\mathrm{M}_{\ell}(\mathrm{x})|p\rangle & =\frac{e^{i \phi_{0} / 2-n_{0} / 4}}{2^{\ell}} \sqrt{\frac{n_{0}^{\ell}}{\ell !}} \sum_{\nu=-\infty}^{\infty} I_{\nu}\left(i \phi_{0} / 2-n_{0} / 4\right) \\
& \times \sum_{n=0}^{\ell}\left(\begin{array}{l}
\ell \\
n
\end{array}\right)\left|p+2 \hbar k_{\mathrm{L}} \nu+(\ell-2 n) \hbar k_{\mathrm{L}}\right\rangle
\end{aligned}
$$

as follows from Eq. (9). Apart from the conditional diffraction by amplitude modulation, the binomial sum accounts for the coherent transfer of photon recoils in units of $\hbar k_{\mathrm{L}}$. With each absorption event the momentum state splits coherently into two branches shifted by $\pm \hbar k_{\mathrm{L}}$, a particular quantum analogue of a Galton board. Note however that the present model differs from the well-known quantum random-walk realizations of a Galton board found in the literature [44 47]. There each step is described by a unitary transformation conditioned on internal qubit states.

\section{Phase-space description}

The theory of center-of-mass interferometry is conveniently carried out in the Wigner-Weyl phase-space representation; its merits were repeatedly demonstrated in the context of near-field interferometry [16, 25, 26, 36, 40, 43, 48]. Here, we provide the phase-space counterparts of the state transformation (5).

Given a matter-wave state $\rho$ prior to the grating, the Wigner function is defined as

$$
w(x, p)=\frac{1}{2 \pi \hbar} \int d s e^{i p s / \hbar}\left\langle x-\frac{s}{2}|\rho| x+\frac{s}{2}\right\rangle .
$$

The conditional, norm-reducing state transformation $\rho \rightarrow \mathbf{M}_{\ell} \rho \mathbf{M}_{\ell}^{\dagger}$ translates into a convolution in phase-space,

$$
w(x, p) \rightarrow w(x, p ; \ell):=\int d p_{0} w\left(x, p-p_{0}\right) T_{\mathrm{L}}\left(x, p_{0} ; \ell\right) .
$$

The convolution kernel for a given absorption number $\ell$ reads

$$
\begin{aligned}
T_{\mathrm{L}}(x, p ; \ell) & =\frac{1}{2 \pi \hbar} \int d s e^{i p s / \hbar} M_{\ell}\left(x-\frac{s}{2}\right) M_{\ell}\left(x+\frac{s}{2}\right) \\
& =\frac{1}{2 \pi \hbar} \sum_{j=-\infty}^{\infty} e^{2 \pi i j x / d} \int d s e^{i p s / \hbar} B_{j}\left(\frac{s}{d} ; \ell\right) .
\end{aligned}
$$

The second expression is a Fourier expansion with $d=$ $\lambda_{\mathrm{L}} / 2=\pi / k_{\mathrm{L}}$ the grating period. Note that the so defined conditional Wigner function is normalized to the absorption probability $\int d x d p w(x, p ; \ell)=P_{\ell}(\rho)$.

The Fourier components $B_{j}(s / d ; \ell)$ generalize the Talbot coefficients, determining the interference pattern in matter-wave interferometry 26]. For $\ell=0$ the coefficients are given by the expressions found for photodepletion gratings [40],

$$
\begin{aligned}
B_{j}(\xi ; 0) & =e^{-n_{0} / 2}\left(\frac{\zeta_{\mathrm{coh}}-\zeta_{\mathrm{abs}}}{\zeta_{\mathrm{coh}}+\zeta_{\mathrm{abs}}}\right)^{j / 2} \\
& \times J_{j}\left(\operatorname{sgn}\left(\zeta_{\mathrm{coh}}+\zeta_{\mathrm{abs}}\right) \sqrt{\zeta_{\mathrm{coh}}^{2}-\zeta_{\mathrm{abs}}^{2}}\right) .
\end{aligned}
$$

Here, the parameters

$$
\zeta_{\mathrm{abs}}(\xi)=\frac{n_{0}}{2} \cos (\pi \xi) \text { and } \zeta_{\mathrm{coh}}(\xi)=\phi_{0} \sin (\pi \xi)
$$

relate to the photon absorption and the dipole interaction, respectively. For $\ell \neq 0$ the conditional Talbot coefficients are

$$
\begin{aligned}
B_{j}(\xi ; \ell) & =\sum_{n=0}^{\ell} \sum_{r=0}^{n}\left(\frac{n_{0}}{4}\right)^{n} \frac{\zeta_{\mathrm{abs}}^{\ell-n}(\xi)}{r !(n-r) !(\ell-n) !} \\
& \times B_{j-n+2 r}(\xi ; 0) .
\end{aligned}
$$

When the detector is insensitive to the internal molecular state one must resort to the unconditional state transformation, i.e. sum over all conditional transformations (15), to obtain the unconditional Wigner function $w^{\prime}(x, p)=\sum_{\ell=0}^{\infty} w(x, p ; \ell)$. This is equivalent to summing over the conditional Talbot coefficients in (16), $B_{j}(\xi)=\sum_{\ell=0}^{\infty} B_{j}(\xi ; \ell)$. After rearranging the terms in (19) and substituting $n$ with $m=2 r-n$ we recognize a series representation of the modified Bessel function, $I_{\nu}(z)=\sum_{k=0}^{\infty}(z / 2)^{2 k+\nu} / k !(\nu+k)$ !. The resulting expression can be simplified further with help of Neumann's addition theorem, $\sum_{j=-\infty}^{\infty} I_{j-\nu}(u) I_{j}(v)=$ $I_{\nu}(u+v)$, and a special case of Graf's addition theorem $\sum_{j=-\infty}^{\infty} J_{j}(u) I_{j+n}(v)=[(u-v) /(u+v)]^{n / 2} J_{-n}(\operatorname{sgn}(u+$ $v) \sqrt{u^{2}-v^{2}}$ [49. Finally, we get the unconditional Talbot coefficients [9, 43]

$$
\begin{aligned}
B_{j}(\xi) & =e^{-\zeta_{\mathrm{abs}}^{\prime}}\left(\frac{\zeta_{\mathrm{coh}}+\zeta_{\mathrm{abs}}^{\prime}}{\zeta_{\mathrm{coh}}-\zeta_{\mathrm{abs}}^{\prime}}\right)^{j / 2} \\
& \times J_{j}\left(\operatorname{sgn}\left(\zeta_{\mathrm{coh}}-\zeta_{\mathrm{abs}}^{\prime}\right) \sqrt{\zeta_{\mathrm{coh}}^{2}-\left(\zeta_{\mathrm{abs}}^{\prime}\right)^{2}}\right)
\end{aligned}
$$


with $\zeta_{\text {abs }}^{\prime}=n_{0} \sin ^{2}(\pi \xi / 2)$.

The conditional expression (19) applies if the molecules or nanoparticles are detected selectively according to their absorption number $\ell$. Otherwise, the expression (20) applies. We note that this expression resembles an earlier model [26], where photon absorption was implemented as a classical random walk in phase space, disregarding the coherent recoil transfer in a standing wave. Surprisingly, the difference merely amounts to a sign flip $\zeta_{\mathrm{coh}} \rightarrow-\zeta_{\mathrm{coh}}$ in (20), which is equivalent to replacing $B_{j}(\xi)$ with $B_{j}(-\xi)=B_{-j}(\xi)$, as follows from the identity

$$
\left(\frac{y-x}{y+x}\right)^{j / 2} \operatorname{sgn}(y+x)^{j}=\left(\frac{y+x}{y-x}\right)^{-j / 2} \operatorname{sgn}(y-x)^{j},
$$

where $x, y \in \mathbb{R}$. Hence, the difference between the two models disappears in the two extreme cases of no absorption, $n_{0} \ll \phi_{0}$, and dominant absorption, $n_{0} \gg \phi_{0}$.

\section{ABSORPTION EFFECTS ON INTERFERENCE}

The results of the previous section can be readily applied to assess the effect of absorption in arbitrary matter-wave diffraction experiments with nanoparticles at optical standing waves. Here we apply our model to two exemplary settings: The Kapitza-Dirac-TalbotLau near-field matter-wave interferometer (KDTLI) and far-field diffraction at a single standing-wave grating. In both cases we explore the influence of absorption and evaluate the predicted interferograms. It turns out that the predictions of the model are fully captured only in the near field.

\section{A. Talbot-Lau near-field interferometer}

In the KDTLI setting, an initially incoherent beam of molecules passes through three equidistantly separated gratings with the same period $d$; first a material grating (G1), then the standing-wave laser grating (G2), and finally another material grating (G3). Molecular matter waves emerging from each source slit at G1 obtain sufficient spatial coherence by propagating the distance $L$ to G2, where they are diffracted. Talbot-Lau interference [50] may then yield a high-contrast fringe pattern of the period $d$ at a distance $L$ further downstream. G3 serves as a movable mask to scan the interference pattern by counting the number of transmitted particles as a function of the lateral shift $x_{\mathrm{s}}$ of G3 relative to G1 and G2. The two material masks have the same opening fraction, i.e. the same ratio $f$ between slit opening and grating period.

For a detailed theoretical derivation of the predicted Talbot-Lau interference signal, we refer the reader to previous publications [25, 26, 43, 48]. The detected signal can be expressed much like in the case of a coherent grating transformation by means of the Talbot coefficients (20)

$$
S\left(x_{\mathrm{s}}\right)=\sum_{j=-\infty}^{\infty} f^{2} \operatorname{sinc}^{2}(j \pi f) B_{2 j}\left(j \frac{L}{L_{\mathrm{T}}}\right) e^{2 \pi i j x_{\mathrm{s}} / d} .
$$

The Talbot length $L_{\mathrm{T}}=d^{2} / \lambda_{\mathrm{dB}}$, including the de Broglie wavelength $\lambda_{\mathrm{dB}}$ of a molecule, appears as the natural unit for the distance between the gratings.

A robust way to quantify the fringe contrast is to fit a sine curve of period $d$ to the noisy measurement data. The ratio between amplitude and offset, i.e. the sine visibility, corresponds to the ratio of the first and the zeroth Fourier component in (22),

$$
\mathcal{V}_{\sin }\left(\frac{L}{L_{\mathrm{T}}}\right)=2 \operatorname{sinc}^{2}(\pi f) B_{2}\left(\frac{L}{L_{\mathrm{T}}}\right) .
$$

Negative values indicate a phase-flipped interference pattern. In the envisaged KDTLI setup, the sinusoidal visibility is close to the visibility $\mathcal{V}=\left(S_{\max }-S_{\min }\right) /\left(S_{\max }+\right.$ $\left.S_{\text {min }}\right)$ defined in terms of the interference minima and maxima.

Figure1(a) compares the expected visibilities for moderate absorption $\left(n_{0}=1\right.$, grey-shaded area) with those for no absorption $\left(n_{0}=0\right.$, solid blue line), both at $\phi_{0}=\pi$. One observes that, compared to the case of a pure phase modulation at G2, absorption decreases the unconditional visibility almost everywhere. This unconditional interferogram results from the incoherent overlay of the conditional interferograms labeled by $\ell$; some of these are phase-flipped with respect to the others resulting in the negative visibilities depicted in Panel (b). In addition in the absence of photon absorptions, the visibility is periodic in the grating separation $L$ with period $L_{\mathrm{T}}$ (solid line in Fig. 11(a)). Absorption breaks this symmetry and doubles the period to $2 L_{\mathrm{T}}$. The reason is that the photon absorption comes with a recoil transfer in units of half the grating momentum, $\hbar k_{\mathrm{L}}$.

Curiously, our measurement-based model for absorption predicts visibilities that look like a mirror image of those from a classical random-walk model (red dashed line in Fig. 1(a)). This difference was not observed in previous experiments [26, 32] because it only shows up in interference patterns recorded with a sufficiently narrow velocity distribution. Recent experiments with improved velocity selection 9] reveal the model discrepancy and provide evidence for the present quantum model.

The unconditional fringe signal (22) underlying the grey-shaded area in Fig. 1(a) and (b) is a sum of conditional interferograms,

$$
S\left(x_{\mathrm{s}} ; \ell\right)=\sum_{j} f^{2} \operatorname{sinc}^{2}(j \pi f) B_{2 j}\left(j \frac{L}{L_{\mathrm{T}}} ; \ell\right) e^{2 \pi i j x_{\mathrm{s}} / d} .
$$

A molecule detector sensitive to the internal state would be able to resolve these interferograms. Their individual 


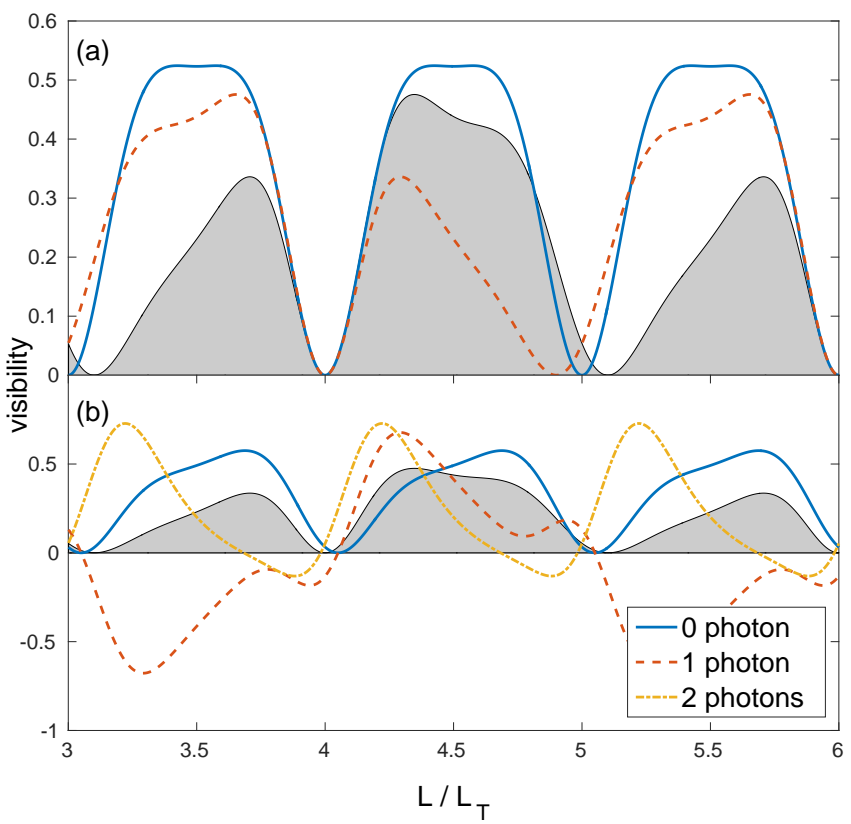

FIG. 1. (a) Sinusoidal visibility (23) of KDTLI as a function of the Talbot parameter $L / L_{\mathrm{T}}$, i.e. the grating distance over Talbot length $L_{\mathrm{T}}$. The case of a pure phase grating (solid blue line, $\phi_{0}=\pi$ at $\left.\sigma_{\mathrm{abs}}=0\right)$ is compared to an absorptive molecule with $n_{0}=1$ (shaded area). A classical random-walk description of absorption (dashed red line) does not match the correct quantum prediction. (b) Visibilities (25) of the constituent conditional interferograms corresponding to $\ell=0,1$, and 2 photon absorptions (solid, dashed, and dash-dotted lines, respectively). The shaded area represents the unconditional visibility as in panel (a). The opening fraction is $f=0.42$ both plots.

sinusoidal visibilities are given by

$$
\mathcal{V}_{\sin }\left(\frac{L}{L_{\mathrm{T}}} ; \ell\right)=2 \operatorname{sinc}^{2}(\pi f) \frac{B_{2}\left(L / L_{\mathrm{T}} ; \ell\right)}{B_{0}(0 ; \ell)}
$$

They can reach as high values as 70\%, see Fig. 1(b).

In Fig. 2(a) and (b) we show cascades of conditional fringe patterns (24) as a function of the G3 shift $x_{\mathrm{s}}$ for the same parameters as before $\left(n_{0}=1, \phi_{0}=\pi, f=0.42\right)$. The panels (a) and (b) correspond to fixed Talbot parameters $L / L_{\mathrm{T}}=3.25$ and 4.25, respectively. All conditional patterns (stacked thin lines) have the same period $d$ as the unconditional signal (thick top line), but the odd absorption numbers can be phase-flipped with respect to the even ones when the Talbot parameter $L / L_{\mathrm{T}}$ is in the range between an odd and a next even integer. This is the case in panel (a) which thus features a lower unconditional contrast than panel (b).

The relative weights of the constituent interferograms, i.e. the transmission probabilities for molecules of given absorption numbers $\ell$, depend on the average absorption strength $n_{0}$. They are given by the mean value of (24)

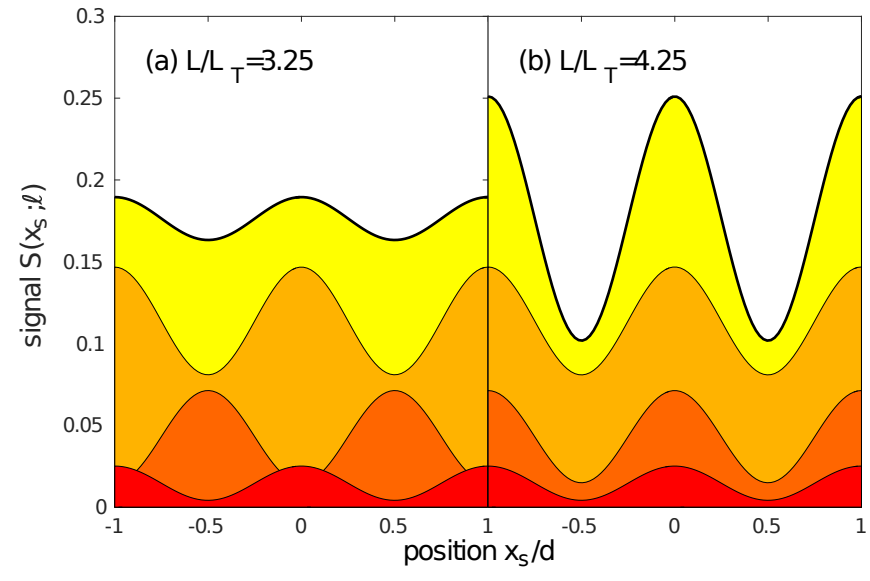

FIG. 2. Panel (a) and (b) show the predicted conditional, $\ell$-dependent fringe patterns (24) underlying the visibilities in Fig. 11 They are plotted as a function of the lateral shift $x_{\mathrm{s}}$ of the third grating at two fixed Talbot parameters $L / L_{\mathrm{T}}=$ 3.25 and 4.25. Each panel contains the stacked conditional interferograms for molecules absorbing $\ell=0,1$, and 2 photons (thin lines, lower mean signal for greater $\ell$-values), as well as the weighted sum over all $\ell$ (unconditional interferogram, thick line on top).

with respect to $x_{\mathrm{s}}$,

$$
\begin{aligned}
\bar{S}_{\ell} & =f^{2} B_{0}(0 ; \ell)=f^{2} e^{-n_{0} / 2}\left(\frac{n_{0}}{2}\right)^{\ell} \\
& \times \sum_{n=0}^{\ell} \sum_{r=0}^{n} \frac{I_{2 r-n}\left(-n_{0} / 2\right)}{2^{n} r !(n-r) !(\ell-n) !} .
\end{aligned}
$$

For the case of $n_{0}=1$, illustrated in Fig. 2] we find that the relative weights of the conditional interferograms decrease from $64 \%$ to $24 \%$ and $8 \%$, for $\ell=0$, 1 , and 2 , respectively. The remaining $4 \%$ of higher absorption numbers are hardly relevant.

\section{B. Far-field interferometry}

Let us now turn to the influence of photon absorption on far-field diffraction at a laser grating [6]. A simple setup is sketched in Fig. 3. We consider a beam of molecules diverging from a point-like source and collimated by a slit aperture of width $D$ at distance $L$ from the source. The laser grating with period $d=\lambda_{\mathrm{L}} / 2$ is placed immediately behind the aperture. Molecular matter waves are diffracted and their density distribution far from the grating exhibits a characteristic interference fringe pattern. For simplicity, we consider here a symmetric arrangement with equal distances between source, grating, and detection plane, as well as a monochromatic beam of molecules at sufficiently high forward velocity $v_{z}$ to allow for a one-dimensional phase-space treatment of the Fresnel-Kirchhoff diffraction integral in the paraxial approximation [51]. 


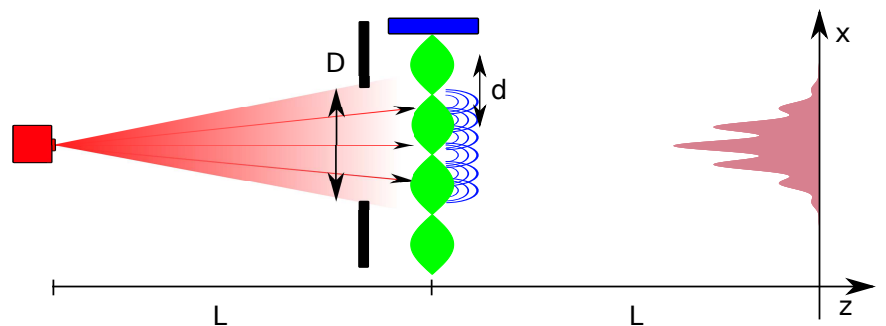

FIG. 3. Sketch of a symmetric far-field configuration for molecular diffraction at a standing-wave grating. A collimated beam of molecules, as produced by an incoherent pointlike source in combination with a collimation slit of width $D$ at distance $L$, is diffracted at a standing laser wave with grating period $d$. The resulting far-field interference pattern can be recorded by a spatially resolving detector in distance $L$ to the laser grating.

We begin with an idealized molecular point source as presented by the (unnormalized) initial Wigner function $w(x, p)=\delta(x)$. After free propagation by the distance $L$ described by the shearing transformation $w(x, p) \rightarrow$ $w\left(x-p L / m v_{z}, p\right)$, the molecular beam is collimated by passing an aperture of width $D$. In phase space, this process is described by a convolution analogous to the grating transformation (15),

$$
\begin{aligned}
w(x, p) \rightarrow & \int_{-\infty}^{\infty} d p_{0} w\left(x, p-p_{0}\right) \Theta\left(|x|-\frac{D}{2}\right) \\
& \times \frac{\sin \left[(2|x|-D) p_{0} / \hbar\right]}{\pi p_{0}}
\end{aligned}
$$

where $\Theta$ is the Heaviside step function. The subsequent grating transformation (15) followed by a further shearing transformation associated with the free propagation to the detector gives the conditional spatial density distribution $w(x ; \ell)=\int d p w(x, p ; \ell)$ on the screen,

$$
\begin{aligned}
w(x ; \ell) & =\frac{d}{D \Delta x} \sum_{j=-\infty}^{\infty} \int_{-D / d}^{D / d} d q e^{2 \pi i q x / \Delta x} \\
& \times B_{j}(q ; \ell) \frac{\sin [\pi(D / d-|q|)(j-2 q d / \Delta x)]}{j-2 q d / \Delta x} .
\end{aligned}
$$

Here, $\Delta x$ denotes the distance between neighboring diffraction peaks on the screen plane,

$$
\Delta x=\frac{h}{d} \frac{L}{m v_{z}}=d \frac{L}{L_{\mathrm{T}}} .
$$

Once again, the unconditional result is obtained by replacing the Talbot coefficients in (28) with (20).

We note that Eq. (28) can be equivalently expressed as

$$
w(x ; \ell)=\frac{d}{D \Delta x}\left|\int_{-\infty}^{\infty} d q e^{2 \pi i q(x-d q) / \Delta x} t_{\ell}(d q)\right|^{2},
$$
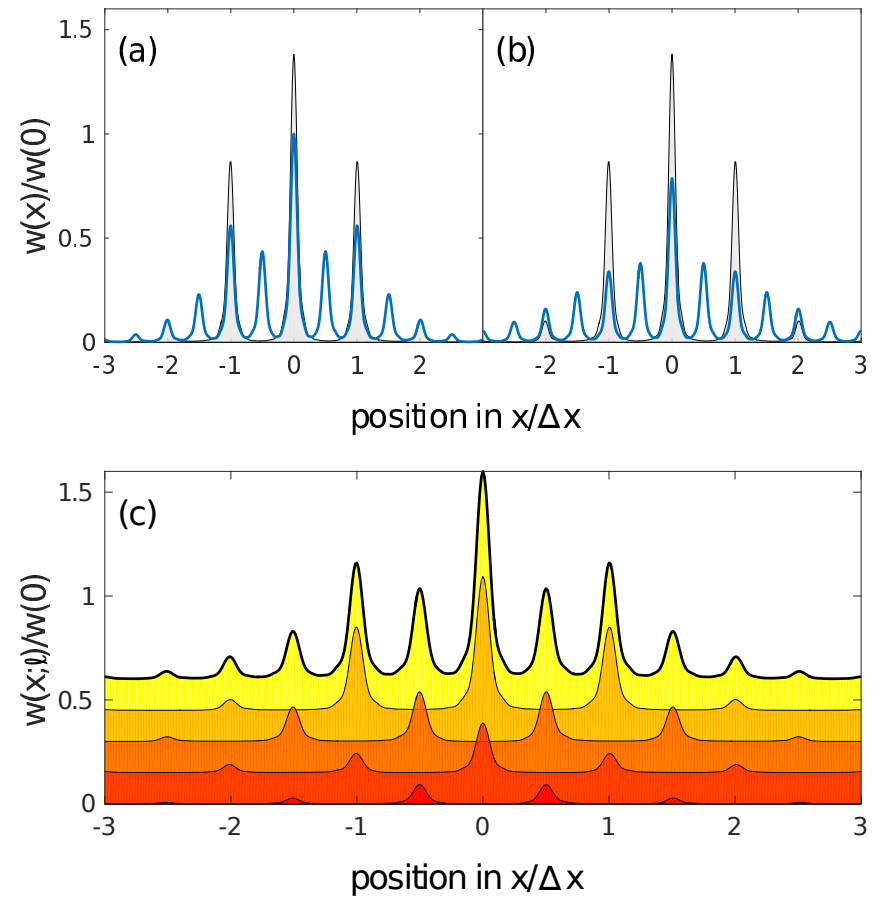

FIG. 4. Far-field interferograms on the screen plane behind a laser grating for non-absorbing (shaded area) and for absorbing (solid line) molecules, assuming state-indiscriminate detection. Panel (a) and (b) correspond to two different absorption strengths, $n_{0}=2$ and $n_{0}=10$, respectively. The shaded curves represent diffraction at a pure phase grating $\left(n_{0}=0\right)$ at $\phi_{0}=2.5$. The screen coordinate is given in units of the expected separations $\Delta x$ of the coherent diffraction maxima. (c) Conditional far-field interferograms contributing to the unconditional fringe signal in Panel (a); the curves are shifted vertically for better illustration. The thin lines from top to bottom are the result of evaluating (28) for $\ell=0,1,2$ photon absorptions, respectively. The thick line represents the unconditional result depicted in Panel (a), i.e. the incoherent sum over all contributions. For this plot, we assume a finite detector resolution of $0.1 \Delta x$ and a collimator width of $D / d=10$.

where the function $t_{\ell}(x)=\Theta(|x|-D / 2) M_{\ell}(x)$ describes the conditional state transformation due to a collimator and an optical grating. Equation (30) has the form of a Kirchhoff integral within the Fresnel approximation [51].

The effect of absorption on far-field interferograms is illustrated in Fig. 4 We present the expected density distribution on the screen with and without absorption. In Panels (a) and (b), the shaded areas stand for a pure phase-grating, i.e. for molecules with a vanishing absorption cross section $\left(n_{0}=0\right)$, whereas the solid line corresponds to the unconditional interferogram of strongly absorbing molecules with $n_{0}=2$ (left) and $n_{0}=10$ (right). In all cases the phase modulation is assumed to be $\phi_{0}=2.5$. One observes that the coherent diffraction peaks at integer multiples of $\Delta x$ get reduced if there is a finite absorption probability, while density peaks at half integer multiples of $\Delta x$ get populated. This is again 
related to the recoil momentum upon absorption of half a grating momentum, as becomes apparent in Fig. 4(c), where the unconditional result (thick line, $n_{0}=2$ ) is decomposed into its components (28) representing the conditional interferograms for fixed absorption numbers $\ell=0,1,2$ (thin lines from top to bottom). Odd absorption numbers are responsible for the additional peaks as they have their diffraction peaks only at odd halves of $\Delta x$.

We note that a classical random-walk model for absorption produces far-field interferograms that are almost identical to the results plotted in Fig. (4) In fact, both models give indistinguishable predictions for Fraunhofer diffraction. This can be seen by carrying out the Fraunhofer far-field approximation $d / \Delta x \ll 1$ in (28),

$$
\begin{aligned}
w(x ; \ell) & \simeq \sum_{j=-\infty}^{\infty} \int_{-D / d}^{D / d} d q e^{2 \pi i q x / \Delta x} B_{j}(q ; \ell) \\
& \times \frac{\sin [\pi(D / d-|q|) j]}{j} .
\end{aligned}
$$

This expression is invariant under the sign flip $j \rightarrow-j$, so that there is no difference between both models in the far-field limit, see Sect. IIC This means that the coherence in the photon momentum transfer in a standingwave grating can only be observed in the near field. The KDTLI setup with sufficiently absorptive molecules, where the laser is neither a pure phase grating nor purely absorptive, is well suited for this purpose [9].

\section{DYNAMICAL DESCRIPTION OF THE OPTICAL GRATING}

In this section, we present a dynamical description of the interplay between the center-of mass-motion and the internal state evolution of a molecule interacting with a standing laser wave. First we introduce a ladder model for the photon absorption of molecules whose internal degrees of freedom act as an effective heat sink. The resulting master equation for the center-of-mass state of the molecule will be found to corroborate the measurementbased model for absorption given in Sect. [II. The model is then generalized to include state-dependent internal properties, which is potentially relevant in experimental scenarios involving highly absorptive nanoparticles.

Finally, our phenomenological treatment of incoherent absorption will be compared to an effective three-level Rabi model for the molecule-light interaction with a finite degree of coherence. It shows that if Rabi oscillations occur they can have a significant impact on the interference pattern.

\section{A. Effective ladder model for absorbing particles}

We consider the following simple absorption model for particles that can absorb several photons without re- emission and whose only known properties are the polarizability and the absorption cross-section: Starting from a particle in its internal ground state $|0\rangle$, every subsequent photon absorption shall excite the internal state to distinct orthogonal states $|0\rangle \rightarrow|1\rangle \rightarrow|2\rangle$. . of increasing internal energies $E_{\ell}=E_{0}+\ell \hbar \omega$. The total state during the interaction with the light field is then described by the time-dependent density matrix $\left\langle x, \ell|\rho| x^{\prime}, \ell^{\prime}\right\rangle$. The goal is to find expressions for the conditional and the unconditional states after the interaction, i.e. for the projections $\rho_{\ell \ell}\left(x, x^{\prime} ; t\right):=\left\langle x, \ell|\rho| x^{\prime}, \ell\right\rangle$ and for the reduced center-of-mass operator $\rho\left(x, x^{\prime} ; t\right)=\sum_{\ell=0}^{\infty} \rho_{\ell \ell}\left(x, x^{\prime} ; t\right)$.

In a one-dimensional description, the particle interacts with the laser while it crosses the Gaussian intensity profile (11) at a fixed longitudinal velocity $v_{z}$. This results in a mean interaction time $t_{\mathrm{L}}=\sqrt{\pi / 2} w_{z} / v_{z}$ and in a time-dependent Hamiltonian

$$
\mathrm{H}=\sum_{\ell=0}^{\infty}\left[E_{\ell}+V_{\ell}(\mathrm{x}, t)\right]|\ell\rangle\langle\ell| .
$$

The kinetic energy term is omitted since we are neglecting the transverse motion of the particle during its passage through the laser grating, see Sect. [II The $\ell$-dependence of the dipole interaction potential takes into account that the particle's polarizability will in general depend on its internal state.

Photon absorption can be described as a random jump process in terms of a Lindblad-type master equation [20, 52], with the jump rate set by the timedependent absorption rate at the antinodes, $\gamma_{\ell}(t)=$ $8 \sigma_{\mathrm{abs}, \ell} P / \pi w_{y} w_{z} \hbar \omega_{\mathrm{L}} \exp \left(-2\left(v_{z} t\right)^{2} / \omega_{z}^{2}\right)$, see Eq. (8). In general, the absorption cross-section may depend on the internal state. When an absorption event occurs, two effects must be considered: an excitation of the internal state up the ladder, $\ell \rightarrow \ell+1$, and the coherent transfer of photon recoil from the standing wave to the particle. Both effects can be implemented by introducing the Lindblad operator

$$
\mathrm{L}=\sum_{\ell=0}^{\infty} \sqrt{\gamma_{\ell}(t)} \cos \left(k_{\mathrm{L}} \mathrm{x}\right)|\ell+1\rangle\langle\ell|
$$

which correlates the internal and external state of the particle. The evolution of the density operator follows the master equation $\partial_{t} \rho=[\mathrm{H}, \rho] / i \hbar+\mathrm{L} \rho \mathrm{L}^{\dagger}-\left\{\mathrm{L}^{\dagger} \mathrm{L}, \rho\right\} / 2$. Expanded in the basis of internal states, we are left with a sequence of coupled ordinary differential equations that are diagonal in position representation. The internally diagonal terms $\rho_{\ell \ell}\left(x, x^{\prime} ; t\right)$ of interest decouple from the 
rest and yield a closed set of equations,

$$
\begin{aligned}
\partial_{t} \rho_{00} & =\left[\frac{V_{0}(x, t)-V_{0}\left(x^{\prime}, t\right)}{i \hbar}-\gamma_{0}(t)\right. \\
& \left.\times \frac{\cos ^{2}\left(k_{\mathrm{L}} x\right)+\cos ^{2}\left(k_{\mathrm{L}} x^{\prime}\right)}{2}\right] \rho_{00}, \\
\partial_{t} \rho_{\ell \ell} & =\left[\frac{V_{\ell}(x, t)-V_{\ell}\left(x^{\prime}, t\right)}{i \hbar}-\gamma_{\ell}(t)\right. \\
& \left.\times \frac{\cos ^{2}\left(k_{\mathrm{L}} x\right)+\cos ^{2}\left(k_{\mathrm{L}} x^{\prime}\right)}{2}\right] \rho_{\ell \ell}+\gamma_{\ell-1}(t) \\
& \times \cos \left(k_{\mathrm{L}} x\right) \cos \left(k_{\mathrm{L}} x^{\prime}\right) \rho_{\ell-1 \ell-1} .
\end{aligned}
$$

The imaginary terms represent the coherent phase modulation due to the dipole interaction, while the other terms describe the redistribution of internal state populations according to the rates $\gamma_{\ell}(t)$. The redistribution is Poissonian if all rates are equal. The first equation can be integrated directly, and the remaining sequence of equations can then be solved successively starting from the initial condition $\rho_{\ell \ell}\left(x, x^{\prime} ;-\infty\right)=\widetilde{\rho}\left(x, x^{\prime}\right) \delta_{\ell, 0}$.

In the simple case of a state-independent dipole potential $V(x, t)$ and absorption rate $\gamma_{0}(t)$, the outgoing solution can be written in compact form,

$$
\rho_{\ell \ell}\left(x, x^{\prime}, \infty\right)=M_{\ell}(x) M_{\ell}\left(x^{\prime}\right) \widetilde{\rho}\left(x, x^{\prime}\right) .
$$

This reproduces our measurement-based result $M_{\ell}(x)$ defined as (9) and $n_{0}=\int d t \gamma_{0}(t)$.

\section{B. Generalized model}

We proceed to generalize the ladder model to the case where the molecular parameters switch to a fixed excitedstate value after the absorption of at least one photon. For this, we introduce dimensionless parameters $\eta_{\mathrm{p}}$ and $\eta_{\mathrm{a}}$ describing the changed excited-state dipole potential and absorption rate, $V_{\ell}=\eta_{\mathrm{p}} V_{0}$ and $\gamma_{\ell}=\eta_{\mathrm{a}} \gamma_{0}$ for all $\ell>0$. Models with a stepwise increase of the polarizability and absorption rate have been employed for the determination of molecular excited-state properties [39, 53].

For simplicity, we approximate the Gaussian laser envelope by a constant intensity switched on for the effective interaction time $t_{\mathrm{L}}=\sqrt{\pi / 2} \omega_{z} / v_{z}$. This assumption, which leaves the time-integrated parameters $\phi_{0}$ and $n_{0}$ of the Poisson model unchanged, is well justified in the Raman-Nath regime and produces analytical results. The coupled equations (34) and (35) for $\ell>0$ can be evaluated to

$$
\begin{aligned}
\rho_{\ell \ell}\left(x, x^{\prime}, t_{\mathrm{L}}\right) & =\widetilde{\rho}\left(x, x^{\prime}\right) M_{\ell}(x) M_{\ell}\left(x^{\prime}\right) \\
& \times \eta_{\mathrm{a}}^{\ell-1}{ }_{1} F_{1}\left(\ell ; \ell+1 ; z\left(x, x^{\prime}\right)\right),
\end{aligned}
$$

where we introduced the abbreviation

$$
z\left(x, x^{\prime}\right)=i\left(\eta_{\mathrm{p}}-1\right)\left[\phi(x)-\phi\left(x^{\prime}\right)\right]-\frac{\eta_{\mathrm{a}}-1}{2}\left[n(x)+n\left(x^{\prime}\right)\right]
$$
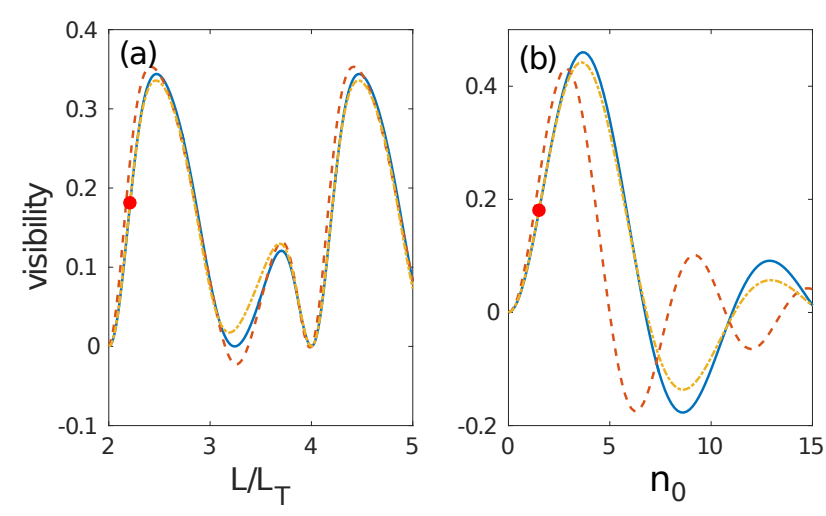

FIG. 5. (a) Sinusoidal visibility as a function of the Talbot parameter $L / L_{\mathrm{T}}$ for molecules with different excitedstate parameters. For the solid curve, we assume that the ground-state values of polarizability and absorption crosssection (corresponding to $n_{0}=1.5$ and $\phi_{0}=1.25 n_{0}$ ) remain the same no matter how many photons are absorbed. The dashed and the dash-dotted curve correspond to an increase of the absorption cross-section and of the polarizability respectively by a factor of 1.5 upon absorption of the first photon. (b) Same visibilities as a function of laser power, i.e. for varying $n_{0}$ at a fixed Talbot parameter of $L / L_{\mathrm{T}}=2.2$. For reference, the red dot marks the same spot $\left(L / L_{\mathrm{T}}, n_{0}\right)$ in both panels. A significant difference between the curves appears at high laser powers.

Here, ${ }_{1} F_{1}$ denotes the confluent hypergeometric function [49]. The solution for $\ell=0$ is identical to the one given in (36).

It is instructive to make use of an integral representation of the hypergeometric function, ${ }_{1} F_{1}(\ell ; \ell+1 ; z)=$ $\ell \int_{0}^{1} d \alpha e^{z \alpha} \alpha^{\ell-1}$, to represent (37) as a conditional postmeasurement state,

$$
\rho_{\ell \ell}=\frac{1}{t_{\mathrm{L}}} \int_{0}^{t_{\mathrm{L}}} d t_{1} \widetilde{\mathrm{M}}_{\ell}\left(t_{1}\right) \widetilde{\rho} \widetilde{\mathrm{M}}_{\ell}^{\dagger}\left(t_{1}\right) .
$$

This allows us to identify generalized measurement operators analogous to (2) and (6),

$$
\begin{aligned}
\widetilde{\mathrm{M}}_{\ell}\left(t_{1}\right) & =\sqrt{\frac{\left(\eta_{\mathrm{a}}\left(1-t_{1} / t_{\mathrm{L}}\right)\right)^{\ell-1} n_{0}^{\ell}}{(\ell-1) !}} \cos ^{\ell}\left(k_{\mathrm{L}} \mathrm{x}\right) \\
& \times \exp \left\{\left(i \phi(\mathrm{x})-\frac{n(\mathrm{x})}{2}\right) \frac{t_{1}}{t_{\mathrm{L}}}\right\} \\
& \times \exp \left\{\left(i \eta_{\mathrm{p}} \phi(\mathrm{x})-\eta_{\mathrm{a}} \frac{n(\mathrm{x})}{2}\right)\left(1-\frac{t_{1}}{t_{\mathrm{L}}}\right)\right\} .
\end{aligned}
$$

They depend on a new parameter $t_{1} \in\left[0, t_{\mathrm{L}}\right]$, which can be interpreted as the time of the first photon absorption. For $t_{1}=t_{\mathrm{L}}$, the operators reduce to their Poissonian counterparts of before.

Figure 5] compares the unconditional visibility (23) of the Poisson model (solid line) to hypothetical cases where the excited-state absorption cross section (dash-dotted 


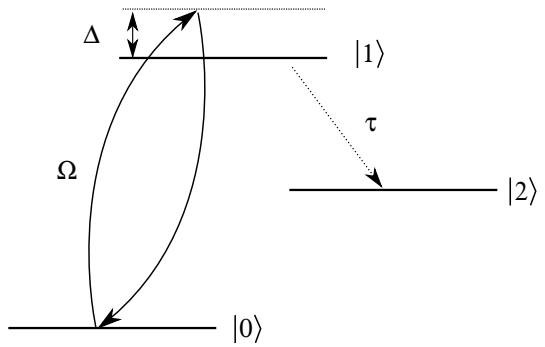

FIG. 6. Scheme of a three-level Rabi model, where the laser drives the transition between the ground state $|0\rangle$ and the excited state $|1\rangle$ at a detuning $\Delta$ off resonance. The excited state with life time $\tau$ can decay without emission into a dark metastable state $|2\rangle$.

line) or the excited-state polarizability (dashed line) are $50 \%$ higher than the ground state values. It turns out that an increased excited-state cross section hardly affects the visibility even though it strongly affects the conditional transmission probability through the grating. The reason is that high-contrast interference is mainly produced by the light-induced phase modulation, and the visibility is therefore more sensitive to absorptioninduced changes of the molecular polarizability. Indeed, we observe a substantial influence of an increased excited state polarizability at high laser power. This might open up a novel spectroscopic application of the KDTLI scheme.

\section{Rabi model for partially coherent absorption}

So far, we have treated photon absorption by molecules incoherently, presuming that electronic transitions are not driven coherently by the light field due to the presence of rapid internal decay channels that involve the excitation of numerous rovibrational degrees of freedom. While this is a good approximation for many large molecules and nanoparticles, one could think of experimental situations [54, 55] that would permit a few coherent Rabi cycles or even the use of Raman transitions, as in atomic beam manipulation [56, 57].

To study the transition from a coherent atom-like description to our model for absorption, we consider the three-level system sketched in Fig. 6. It consists of a ground state $|0\rangle$, an exited state $|1\rangle$ with the life time $\tau$, and a metastable dark state $|2\rangle$. The excited state shall decay exclusively to the dark state without emitting a photon. This Rabi toy model is useful as it can be treated analytically. It is also employed in atomic experiments, e.g. to describe absorptive optical masks [4, 7]. We consider the experimental situation where only ground-state molecules are detected in the end.

We resort once again to a one-dimensional description in the Raman-Nath regime where the transverse motion during the interaction is negligible. The laser shall drive the transition between $|0\rangle$ and $|1\rangle$ with a detuning $\Delta$ relative to the energy difference. The laser-molecule interaction is then characterized by a position-dependent Rabi frequency in the rotating wave approximation [58],

$$
\Omega(x)=-\frac{\mathbf{d}_{01} \cdot \mathbf{E}_{0}}{\hbar} \cos \left(k_{\mathrm{L}} x\right)=: \Omega_{0} \cos \left(k_{\mathrm{L}} x\right),
$$

given the transition dipole moment $\mathbf{d}_{01}$. In a frame rotating at the laser frequency $\omega_{\mathrm{L}}$, the interaction Hamiltonian then reads as $\mathrm{H}=\frac{\hbar}{2}(\Omega(\mathrm{x})|1\rangle\langle 0|-\Delta| 1\rangle\langle 1|)+$ h.c. For the spontaneous decay to the dark state, we introduce the jump operator $\mathrm{L}=|2\rangle\langle 1| / \sqrt{\tau}$, which yields the final master equation $\partial_{t} \rho=[\mathrm{H}, \rho] / i \hbar+\mathrm{L} \rho \mathrm{L}^{\dagger}-\left\{\mathrm{L}^{\dagger} \mathrm{L}, \rho\right\} / 2$. Note that a radiative decay would imply a more complicated decoherence master equation [59].

A decomposition of the density operator into the matrix elements $\rho_{n n^{\prime}}\left(x, x^{\prime} ; t\right):=\left\langle x, n|\rho| x^{\prime}, n^{\prime}\right\rangle$ yields a linear system of nine partially coupled differential equations. The system can be diagonalized exactly, but we will omit the lengthy general solutions and focus on limiting cases.

In the limit of no decay, $\tau \rightarrow \infty$, we obtain the well-known Rabi oscillation between ground and excited state at the position-dependent frequency $\Omega_{R}(x)=$ $\sqrt{\Delta^{2}+\Omega^{2}(x)}[58$. For a finite decay time comparable to the interaction period, the coherences $\rho_{01}$ and $\rho_{10}$ get exponentially suppressed, which leads to a damping of the Rabi oscillations and to a population transfer to the dark state.

In contrast, if the excitation life time is short, $\tau \ll$ $t_{\mathrm{L}}$, the oscillation dies out before a Rabi cycle is completed. Dropping all terms containing the fast damping $\exp \left(-t_{\mathrm{L}} / \tau\right)$ and expanding the eigenfrequencies of the system to lowest order in $\tau / t_{\mathrm{L}}$, the approximate matrix element of the ground-state density operator reads

$$
\begin{aligned}
& \rho_{00}\left(x, x^{\prime}\right) \simeq \\
& \exp \left\{-\frac{1}{2} \frac{t_{\mathrm{L}} \tau \Omega_{0}^{2}}{1+4 \Delta^{2} \tau^{2}}\left[\cos ^{2}\left(k_{\mathrm{L}} x\right)+\cos ^{2}\left(k_{\mathrm{L}} x^{\prime}\right)\right]\right\} \\
& \times \exp \left\{-i \frac{t_{\mathrm{L}} \Delta \tau^{2} \Omega_{0}^{2}}{1+4 \Delta^{2} \tau^{2}}\left[\cos ^{2}\left(k_{\mathrm{L}} x\right)-\cos ^{2}\left(k_{\mathrm{L}} x^{\prime}\right)\right]\right\}
\end{aligned}
$$

We notice that this coincides with the conditional density matrix (36) for zero absorptions from the above ladder model. That is, the molecule acts as an incoherent 1photon absorber in this limit, and we can identify the effective phase shift and mean absorption number parameters by comparison,

$$
\phi_{0} \hat{=}-\frac{t_{\mathrm{L}} \Delta \tau^{2} \Omega_{0}^{2}}{1+4 \Delta^{2} \tau^{2}}, \quad n_{0} \hat{=} \frac{t_{\mathrm{L}} \tau \Omega_{0}^{2}}{1+4 \Delta^{2} \tau^{2}} .
$$

Similar results were derived and discussed in the case of Bragg diffraction [60]. As demonstrated in atomic experiments, a pure phase grating can be realized in the far off-resonant case [30], with $\phi_{0} \approx-t_{\mathrm{L}} \Omega_{0}^{2} / 4 \Delta$, and a pure absorptive grating in the resonant case [4, 7], with $n_{0}=t_{\mathrm{L}} \tau \Omega_{0}^{2}[\underline{[61]}$. 

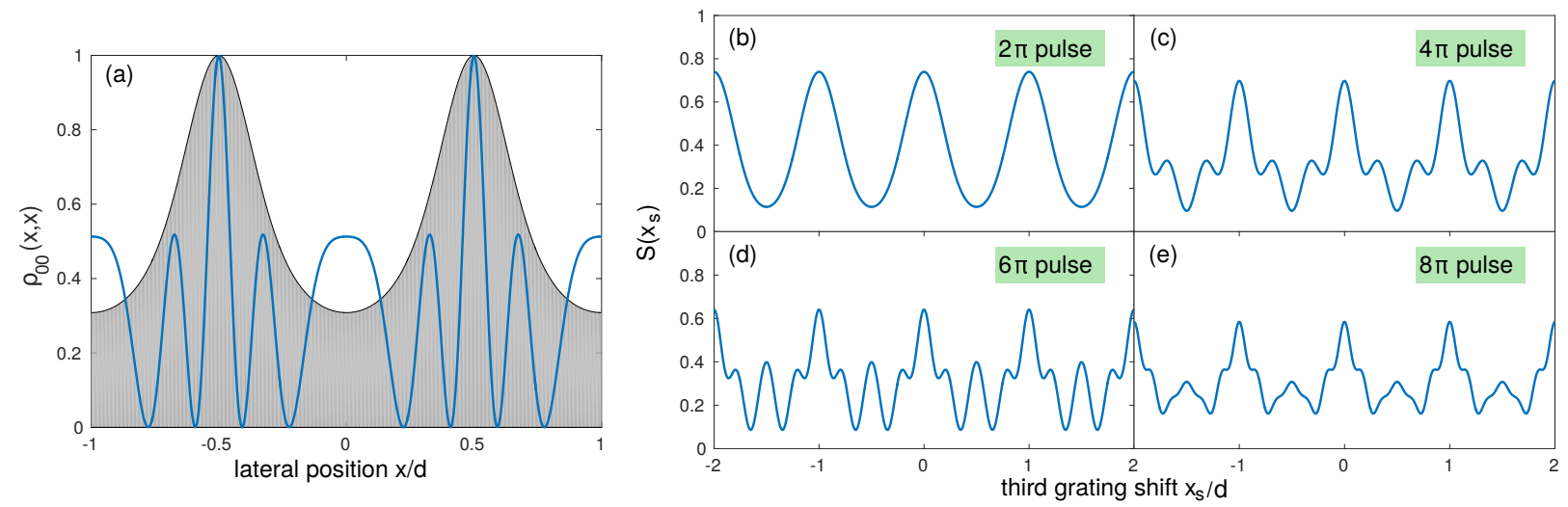

FIG. 7. (a) Probability density for the transmission of ground-state molecules through a standing wave of period $d=\lambda_{\mathrm{L}} / 2$ as a function of position. The shaded area corresponds to the conditional probability for zero absorptions, taken from the ladder model (26) with $n_{0}=1.2$. An evaluation of the coherent three-level Rabi model yields the blue solid line, assuming $\Omega_{0} t_{\mathrm{L}}=4 \pi$ (i.e. a $4 \pi$ pulse length at the antinodes) and a long excited-state life time of $\tau=t_{\mathrm{L}}$. (b)-(e) Numerical results for the KDTLI fringe signal in the presence of molecular Rabi oscillations, according to the three-level model on resonance $\left(\Delta=0, \tau=t_{\mathrm{L}}\right)$. We use a setup with $L=2 L_{T}$ grating separation, and $f=0.1$ opening fraction for G1 and G3; only ground-state molecules are detected. The antinode intensity is chosen such that it amounts to an effective pulse length $\Omega_{0} t_{\mathrm{L}}$ increasing from $2 \pi$ to $8 \pi$ in panels (b) to (e), respectively. We observe higher-order fringes emerging with each Rabi cycle.

When the interaction time is comparable to the decay time of the excited state, the time evolution is governed by several cycles of damped Rabi oscillations, and the transmission of ground-state molecules will depend in an oscillatory fashion on the precise value $t_{\mathrm{L}}$ of the interaction time. We illustrate this in Fig. 77(a), which depicts the position-dependent probability (blue solid line) that a molecule passes a resonant standing laser wave in the ground state. We assume an interaction of $\Omega_{0} t_{\mathrm{L}}=4 \pi$ mimicking a $4 \pi$ pulse at the antinodes, and $\tau=t_{\mathrm{L}}$. This is compared to the conditional transmission probability for zero absorptions in the ladder model using $n_{0}=1.2$ (shaded area). For the Rabi case, one can observe an oscillation of the transmission probability. Here the minima correspond to $\pi$-pulses [58], where all particles are either in the excited or in the dark state. At an antinode, the transmission is below $100 \%$ due to losses into the dark state.

As a consequence of the Rabi features in the laser grating, higher-order fringe oscillations should appear in the molecular near-field interferograms. This is demonstrated for KDTLI in the four interferograms of Fig. [7(b)(e). They were computed by evaluating the ground-state density operator $\rho_{00}\left(x, x^{\prime} ; t_{\mathrm{L}}\right)$ and inserting this solution into expression (24).

This assumes that excited-state molecules decay to the dark state before detection, and that dark-state molecules are not recorded. As before, we consider the resonant situation, $\Delta=0$, and a fairly long life time, $\tau=t_{\mathrm{L}}$. The parameters were chosen such that the light intensity at the antinodes and the interaction time amount to an effective pulse length of one to four full Rabi cycles in panel (b)-(e). Here, we assume the KDTL setup to operate at a grating separation of two Talbot lengths and with a small open fraction $f=0.1$ at G1 and G3, not to wash out higher harmonics in the fringe pattern. The panels clearly show the appearance of these higher harmonics emerging with each additional Rabi cycle during the interaction time.

Higher fringe oscillations may serve to increase the phase sensitivity of near-field interference schemes with standing-wave gratings, thus boosting the precision in potential metrological applications.

\section{CONCLUSIONS AND OUTLOOK}

We have presented a measurement-based model for photon absorption at standing laser waves to describe matter-wave diffraction at laser gratings. The model is particularly well suited for complex molecules and clusters which can dissipate the heat of several light quanta amongst their numerous internal degrees of freedom. We noted a subtle and intricate difference compared to a classical random-walk model for absorption, which goes unnoticed in far-field diffraction. In the near field it can be observed that quantum interference prevails even in the case of significant absorption and state-insensitive particle detection - a consequence of the interplay between coherent phase modulation at the standing-wave potential and a discrete coherent random walk in steps of single photon recoils in momentum space. Only recently, measurements in a near-field KDTLI setup with $\mathrm{C}_{70}$ molecules provided sound experimental evidence for the validity of our measurement-based model [9], which is also corroborated by a dynamical master-equation approach based solely on phenomenological parameters: The dipole polarizability and the absorption cross-section 
of the particle. We also showed that our approach is extendable to the more general case of parameters that depend on the internal state of the particle.

Finally, we studied the impact of coherent Rabi cycles on the absorption behavior of molecules in cases where the photo-induced internal excitation has a sufficiently long life time. We found that Rabi oscillations imprint an additional oscillatory structure onto the particle state upon transmission through a laser grating, which creates higher harmonics in near-field interferograms. This may be relevant for increasing the precision in potential metrological applications.

\section{ACKNOWLEDGMENTS}

We thank M. Arndt, J. P. Cotter, S. Eibenberger, and L. Maierhofer for helpful discussions and the fruitful collaboration. We acknowledge support from the European Commission within NANOQUESTFIT (Contract No. 304886). S.N. is supported by the National Research Foundation, Prime Minister's Office, Singapore and the Ministry of Education, Singapore under the Research Centres of Excellence programme.
[1] A. Bassi, K. Lochan, S. Satin, T. P. Singh, and H. Ulbricht, Rev. Mod. Phys. 85, 471 (2013).

[2] K. Hornberger, S. Gerlich, P. Haslinger, S. Nimmrichter, and M. Arndt, Rev. Mod. Phys. 84, 157 (2012).

[3] S. Eibenberger, X. Cheng, J. P. Cotter, and M. Arndt, Phys. Rev. Lett. 112, 250402 (2014).

[4] R. Abfalterer, C. Keller, S. Bernet, M. K. Oberthaler, J. Schmiedmayer, and A. Zeilinger, Phys. Rev. A 56, R4365 (1997).

[5] M. Arndt, O. Nairz, J. Vos-Andreae, C. Keller, G. Van der Zouw, and A. Zeilinger, nature 401, 680 (1999).

[6] O. Nairz, B. Brezger, M. Arndt, and A. Zeilinger, Phys. Rev. Lett. 87, 160401 (2001).

[7] A. Turlapov, A. Tonyushkin, and T. Sleator, Phys. Rev. A 68, 023408 (2003).

[8] S. Eibenberger, S. Gerlich, M. Arndt, M. Mayor, and J. Tuxen, Phys. Chem. Chem. Phys. 15, 14696 (2013).

[9] J. P. Cotter, S. Eibenberger, L. Mairhofer, X. Cheng, P. Asenbaum, M. Arndt, K. Walter, S. Nimmrichter, and K. Hornberger, Nat. Commun. 6, 7336 (2015).

[10] M. S. Chapman, T. D. Hammond, A. Lenef, J. Schmiedmayer, R. A. Rubenstein, E. Smith, and D. E. Pritchard, Phys. Rev. Lett. 75, 3783 (1995).

[11] M. O. Scully, B.-G. Englert, and H. Walther, Nature 351, 111 (1991).

[12] K. Hornberger, L. Hackermüller, and M. Arndt, Phys. Rev. A 71, 023601 (2005).

[13] D. E. Chang, C. Regal, S. Papp, D. Wilson, J. Ye, O. Painter, H. J. Kimble, and P. Zoller, Proc. Natl. Acad. Sci. USA 107, 1005 (2010).

[14] O. Romero-Isart, M. L. Juan, R. Quidant, and J. I. Cirac, New J. Phys. 12, 033015 (2010).

[15] O. Romero-Isart, A. C. Pflanzer, F. Blaser, R. Kaltenbaek, N. Kiesel, M. Aspelmeyer, and J. I. Cirac, Phys. Rev. Lett. 107, 020405 (2011).

[16] J. Bateman, S. Nimmrichter, K. Hornberger, and H. Ulbricht, Nat. Commun. 5, 4788 (2014).

[17] M. A. Nielsen and I. L. Chuang, Quantum Computation and Quantum Information ((Cambridge University Press, Cambridge, UK, 2011)).

[18] A. Peres, Quantum Theory: Concepts and Methods (Fundamental Theories of Physics) ((Kluwer academic publishers, New York, 1993)).

[19] H. Breuer and F. Petruccione, The Theory of Open Quantum Systems ((Oxford University Press, Oxford, UK,
2002))

[20] H. M. Wiseman and G. J. Milburn, Quantum measurement and control ((Cambridge University Press, Cambridge, UK, 2009)).

[21] M. L. Bellac, Quantum Physics ((Cambridge University Press, Cambridge, UK, 2012)).

[22] I. D. Mayergoyz, Plasmon resonances in nanoparticles ((World Scientific, New Jersey, 2013)).

[23] W. P. Schleich, Quantum Optics in Phase Space, 1st ed. ((Wiley-VCH, Berlin, 2001)).

[24] M. Moharam, T. Gaylord, and R. Magnusson, Opt. Commun. 32, 19 (1980).

[25] S. Nimmrichter and K. Hornberger, Phys. Rev. A 78, 023612 (2008).

[26] K. Hornberger, S. Gerlich, H. Ulbricht, L. Hackermüller, S. Nimmrichter, I. V. Goldt, O. Boltalina, and M. Arndt, New J. Phys. 11, 043032 (2009).

[27] B. A. Stickler and K. Hornberger, Phys. Rev. A 92, 023619 (2015).

[28] D. L. Freimund, K. Aflatooni, and H. Batelaan, Nature 413, 142 (2001).

[29] P. L. Gould, G. A. Ruff, and D. E. Pritchard, Phys. Rev. Lett. 56, 827 (1986).

[30] E. M. Rasel, M. K. Oberthaler, H. Batelaan, J. Schmiedmayer, and A. Zeilinger, Phys. Rev. Lett. 75, 2633 (1995).

[31] R. W. McGowan, D. M. Giltner, and S. A. Lee, Opt. Lett. 20, 2535 (1995).

[32] S. Gerlich, L. Hackermüller, K. Hornberger, A. Stibor, H. Ulbricht, M. Gring, F. Goldfarb, T. Savas, M. Muri, M. Mayor, and M. Arndt, Nat. Phys. 3, 711 (2007).

[33] S. B. Cahn, A. Kumarakrishnan, U. Shim, T. Sleator, P. R. Berman, and B. Dubetsky, Phys. Rev. Lett. 79, 784 (1997).

[34] P. J. Martin, B. G. Oldaker, A. H. Miklich, and D. E. Pritchard, Phys. Rev. Lett. 60, 515 (1988).

[35] H. C. van de Hulst, Light scattering by small particles ((Dover, New York, 1957)).

[36] K. Hornberger, J. E. Sipe, and M. Arndt, Phys. Rev. A 70, 053608 (2004).

[37] P. Busch, M. Grabowski, and P. Lahti, Operational Quantum Physics, Lecture Notes in Physics Monographs ((Springer, Berlin, 2009)).

[38] R. Haufler, L.-S. Wang, L. Chibante, C. Jin, J. Conceicao, Y. Chai, and R. Smalley, Chem. Phys. Lett. 179, 
449 (1991).

[39] F. Henari, J. Callaghan, H. Stiel, W. Blau, and D. Cardin, Chem. Phys. Lett. 199, 144 (1992).

[40] S. Nimmrichter, P. Haslinger, K. Hornberger, and M. Arndt, New J. Phys. 13, 075002 (2011).

[41] P. Haslinger, N. Dorre, P. Geyer, J. Rodewald, S. Nimmrichter, and M. Arndt, Nat. Phys. 9, 144 (2013).

[42] N. Dörre, J. Rodewald, P. Geyer, B. von Issendorff, P. Haslinger, and M. Arndt, Phys. Rev. Lett. 113, 233001 (2014).

[43] S. Nimmrichter, Macroscopic Matter Wave Interferometry ((Springer, Heidelberg, 2014)).

[44] Y. Aharonov, L. Davidovich, and N. Zagury, Phys. Rev. A 48, 1687 (1993).

[45] D. A. Harmin, Phys. Rev. A 56, 232 (1997).

[46] D. Bouwmeester, I. Marzoli, G. P. Karman, W. Schleich, and J. Woerdman, Phys. Rev. A 61, 013410 (1999).

[47] J. Kempe, Contemp. Phys. 44, 307 (2003).

[48] M. Arndt, N. Dörre, S. Eibenberger, P. Haslinger, J. Rodewald, K. Hornberger, S. Nimmrichter, and M. Mayor, Proceedings of the International School of Physics "Enrico Fermi" 188, 89 (2015).

[49] M. Abramowitz and I. A. Stegun, Handbook of Mathematical Functions: with Formulas, Graphs, and Mathematical Tables ((Dover, New York, 1965)).

[50] P. R. Berman, Atom interferometry ((Academic press, San Diego, 1997), 1997).
[51] M. Born and E. Wolf, Principles of Optics: Electromagnetic Theory of Propagation, Interference and Diffraction of Light, 7th ed. ((Cambridge University Press, Cambridge, UK, 1999)).

[52] S. Dyrting and G. J. Milburn, Phys. Rev. A 49, 4180 (1994).

[53] H. Gratz and A. Penzkofer, J. Photoch. Photobio. A 127, 21 (1999).

[54] A. G. Adam, T. E. Gough, N. R. Isenor, and G. Scoles, Phys. Rev. A 32, 1451 (1985).

[55] K. Shimoda, High-Resolution Laser Spectroscopy (Topics in Applied Physics), 1st ed. ((Springer, Berlin, 1976)).

[56] D. Schlippert, J. Hartwig, H. Albers, L. L. Richardson, C. Schubert, A. Roura, W. P. Schleich, W. Ertmer, and E. M. Rasel, Phys. Rev. Lett. 112, 203002 (2014).

[57] K.-Y. Chung, S.-W. Chiow, S. Herrmann, S. Chu, and H. Müller, Phys. Rev. D 80, 016002 (2009).

[58] C. Gerry and P. Knight, Introductory Quantum Optics ((Cambridge University Press, Cambridge, UK, 2004)).

[59] B. W. Shore and P. L. Knight, J. Mod. Optic. 40, 1195 (1993).

[60] D. O. Chudesnikov and V. P. Yakovlev, Laser Phys. 1, 110 (1991).

[61] An additional prefactor of $4 \sqrt{\pi / 2}$ appears in the references for $\phi_{0}$ and $n_{0}$, because of a different definition of the effective electric field amplitude. 